\title{
Sheet resistance and surface topology time dynamics of intrinsic oxide film on InSe crystals
}

\author{
V.M. Katerynchuk, Z.D. Kovalyuk \\ I.M. Frantsevych Institute of Materials Science Problems, NAS of Ukraine, Chernivtsi Department \\ 5, Iryna Vilde str., 58001 Chernivtsi,Ukraine; E-mail: chimsp@ukrpost.ua
}

\begin{abstract}
It has been shown that a result of InSe crystal oxidation is formation of an intrinsic oxide film that has not insulating but conductive properties. This conductive film forms a potential barrier with the semiconductor substrate. Sheet resistance measurements of the InSe oxide film in dependence on the oxidation time under various temperature conditions were carried out. The resistance was also tested for oxide films obtained for two mutually orthogonal crystal faces: perpendicular and parallel to the c axis. It has been established that the film sheet resistance is substantially changed only for $5 \mathrm{~min}$ of the oxidation time, and further oxidation does not affect its value that is about 100-150 Ohm/square. Surface topology of InSe intrinsic oxide was studied using the atomic-force microscopy method. It was found that this surface becomes nanostructured and contains nanoneedles oriented perpendicularly to the plane of sample surface. Dynamics of surface topology changes in dependence on temperature-time conditions of the oxidation process has been ascertained. It manifests itself in a change of lateral and vertical dimensions of nanoneedles as well as their density.
\end{abstract}

Keywords: InSe, thermal oxidation, intrinsic oxide, sheet resistance, atomic-force microscopy, surface topology.

Manuscript received 21.04.10; accepted for publication 02.12.10; published online 28.02.11.

\section{Introduction}

Thermal oxidation of semiconductors is one of the methods to obtain high-quality films of oxides. These oxides can possess different properties of conductivity and optical transmission depending on the type of compound, technological factors and thickness of films. Intrinsic oxides are often used in different types of heterostructures, because they reduce the density of defects at interfaces [1].

In the process of heteroepitaxy the grown new chemical phase forms, as a rule, not a flat but nanostructured surface layer [2]. Nanostructured objects can have different forms and sizes, which is determined by technological factors [3]. As oxidation of semiconductors is one of heteroepitaxy types, then a study of the surface topology inherent to oxides is of great interest.

Time dynamics of the sheet resistance and topology of the oxidized surface were investigated in this work for intrinsic oxide films of InSe crystals.

\section{Experimental}

InSe crystals were grown by the Bridgmann method. Substrates from them were prepared by cleavage of plates from an ingot. In this case, a normal $\mathbf{N}$ to the substrate surface and crystallographic $\mathbf{c}$ axis were collinear. In another case, when $\mathbf{N} \perp \mathbf{c}$, the desired crystal face was at first cut out and then treated by chemical etchants. Thermal oxidation of InSe substrates was carried out in air at temperatures of 350,400 and $450{ }^{\circ} \mathrm{C}$ in an electric furnace with a controlled block of temperature stabilization. The oxidation period ranged from several minutes to several tens of hours.

The obtained films of oxides on $p$-InSe possessed electron-type conductivity and formed a potential barrier with the substrate. The depletion layer of the intrinsic oxide-p-InSe heterojunction served for the insulation of the film from substrate. This allowed to measure the sheet resistance of the oxide films depending on technological conditions for their preparation. Surface topology of non-oxidized and oxidized samples of InSe 
crystals was investigated using an atomic-force microscope (AFM) of the Nanoscope IIIa Dimension 3000 SPM type (Digital Instruments, USA).

\section{Results and discussion}

Fig. 1 shows the sheet resistance $R_{s}$ of the oxide films in dependence on the oxidation time $t$, which is represented in semilogarithmic coordinates. As one can see from the figure, $R_{s}$ changes essentially only for the initial period of oxidation, which did not exceed $5 \mathrm{~min}$. A decrease of $R_{s}$ with time is the higher and the sheet resistance for the initial period of oxidation is the lower the higher temperature of oxidation is. A further increase of oxidation time of the substrate does not cause an essential variation of the resistance, which becomes stabilized at the level of $100 \mathrm{Ohm} / \square$. The tendency of changes of $R_{s}$ for a crystallography plane parallel to the $c$ axis does not differ, in fact, in no way from the case when the plane of oxidation is perpendicular to c. Nevertheless, a more sharp character of the $R_{\mathrm{S}}=f(t)$ dependence indicates a faster process of oxidation on the corresponding crystal face and a less sheet resistance of this oxide film.

The obtained dependences of $R_{s}$ suppose the following qualitative explanation for oxidation of the substrate. Both indium and selenium oxides may be formed on InSe surface with the same probability, because this compound is a binary mixture. However, selenium oxides decompose at temperatures higher than $100{ }^{\circ} \mathrm{C}$ and, therefore, the decomposition components easily evaporate. In our experiment, the oxidation temperature of the substrates was $350-450{ }^{\circ} \mathrm{C}$ and, thereof, it remains to suppose that only indium oxide with a chemical structure close to $\mathrm{In}_{2} \mathrm{O}_{3}$ becomes created. With increasing the thickness of this oxide film, there is a restriction of the access for oxygen to the oxide-semiconductor interface. The same concerns the reverse process of selenium oxide volatilization. Therefore, the most essential changes of the oxide sheet resistance are observed for several initial minutes of substrate oxidation.

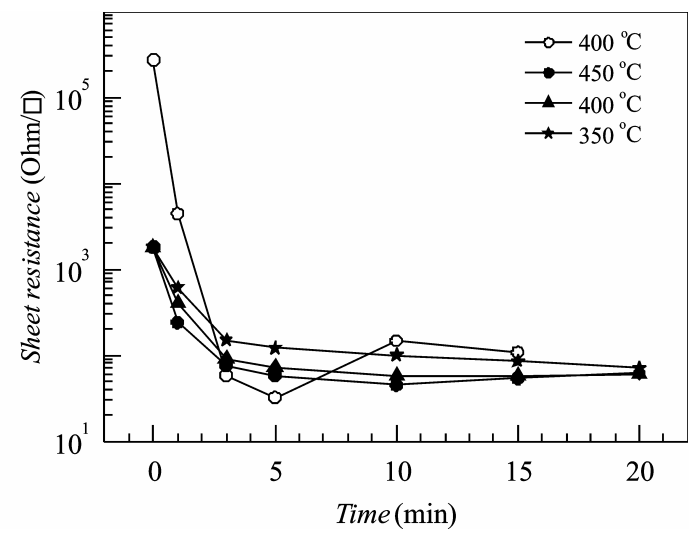

Fig. 1. The dependence of sheet resistance $v s$ temperature and oxidation time for $p$-InSe oxide. Open circles correspond to oxide in $\| \mathbf{c}$ plane; filled ones $-\perp \mathbf{c}$.

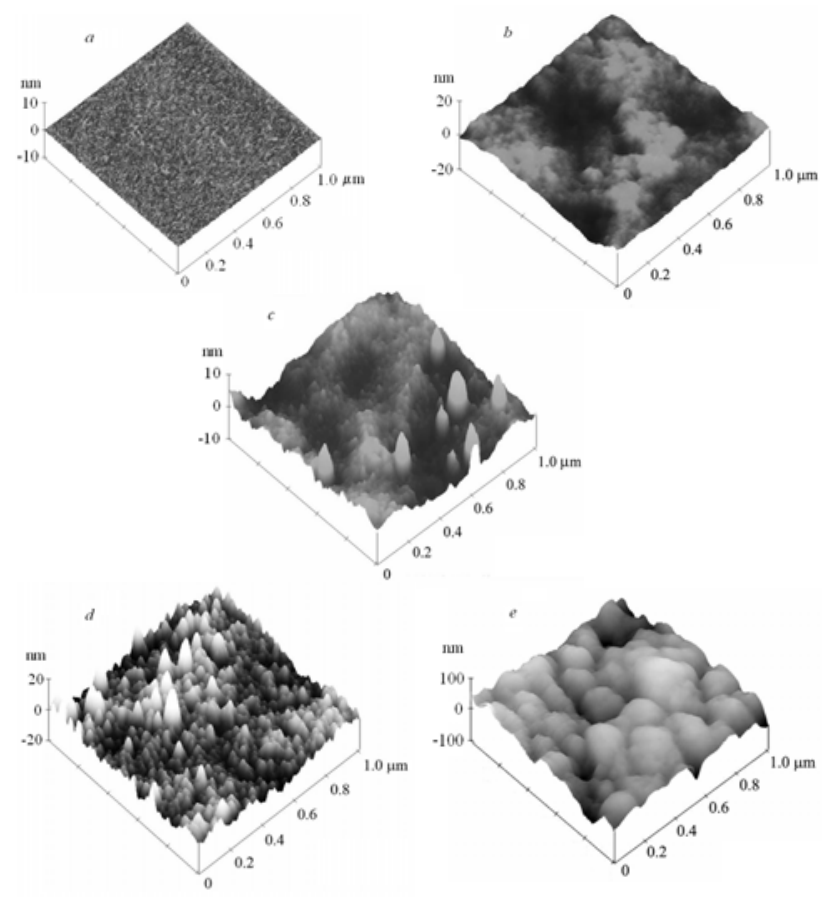

Fig. 2. AFM-images of the surface fragment for InSe (0001) non-oxidized $(a)$ and oxidized $(b, c, d, e)$ crystals at $420^{\circ} \mathrm{C}$ : $b-0.25, c-1, d-5, e-20$ hours of oxidation.

Dynamics of time changes in surface topology inherent to InSe intrinsic oxide films obtained at the temperature $420^{\circ} \mathrm{C}$ is shown in Fig. 2. To make it more pronounced, the process of thermal oxidation of substrates was increased up to $20 \mathrm{~h}$. Fig. 2 a shows a surface fragment of a non-oxidized substrate. This surface is a crystal natural plane after cleaving a sample from the ingot. The arithmetic mean value of the surface roughness $R_{a} \approx 0.053 \mathrm{~nm}$, which indicates high quality of the surface and enables to consider it as atomically smooth. After oxidation of the samples for $15 \mathrm{~min}$, the clusters of oxide crystallites appears on InSe surface, and they are coloured in Fig. $2 b$ in white. They jut out on the surface along with the nanodimensional nonoxidized areas of dark colour. Lateral sizes of the oxide crystallites are about hundred nanometers. The highest height deviation of the crystallite aggregates from the substrate basal plane is close to $7-8 \mathrm{~nm}$, which corresponds to the value of four lattice parameters of InSe $\left(c=1.67 \mathrm{~nm}\right.$ [4]). In this case, the value of $R_{a}$ was increased up to $0.422 \mathrm{~nm}$.

The increase of the oxidation time for the substrate up to $1 \mathrm{~h}$ results in a decrease of lateral sizes in the nonoxidized areas (Fig. 2c). At the same time, a small quantity of oxide crystallites like to nanoneedles appears on the surface, and ordering the surface elements takes place. The height of certain nanoneedles attained $10 \mathrm{~nm}$. A value of $R_{a}$ was about of $0.631 \mathrm{~nm}$, and the height of a prevailing number of the oxide crystallites was increased up to $0.8-2.5 \mathrm{~nm}$.

\section{(C) 2011, V. Lashkaryov Institute of Semiconductor Physics, National Academy of Sciences of Ukraine}


For the oxide obtained at oxidation of the substrate for $5 \mathrm{~h}$, formation of a sufficiently uniform ensemble of the nanoneedles oriented normally to the surface is typical (Fig. 2d). The nanoneedles density attains values of the order of $10^{10}-10^{11} \mathrm{~cm}^{-2}$ and their sizes are different both in height and basis' radius. However, the height of nanoneedles became increased nearly twice in comparison to Fig. 2c. After oxidation of the substrates for $20 \mathrm{~h}$, topology of the oxidized surface changes essentially, and the oxide crystallites take a dome-shaped form (Fig. 2e). This is an evidence that surface topology in the latter case is caused by recrystallization processes. Due to coalescence of the crystallites, creation of the oxide layer with an effective height of nanoformations close to $50 \mathrm{~nm}$ begins.

The revealed regularities of surface topology for oxides prepared under different technological conditions indicate that growing the oxide is not two-dimensional and leads to creation of different nanoformations. Interface mechanical stresses can be reasons for the appearance of these nanoformations at nucleation of the oxide molecules and their subsequent coalescence into shapes corresponding to the maximum thermodynamic stability.

\section{Conclusions}

Time dynamics is ascertained for the sheet resistance and surface topology inherent to the intrinsic oxide layer on InSe crystals. The maximum changes of the oxide film sheet resistance take place at the initial stage of oxidation for times below $5 \mathrm{~min}$. This resistance is about
$100 \mathrm{Ohm} / \square$ and does not essentially change with increasing the oxidation time.

Surface topology of both non-oxidized and oxidized InSe was investigated using the atomic-force microscopy method. For cleaved surfaces of the nonoxidized samples, the surface roughness is close to the atomic one. A characteristic feature of the oxidized surfaces is their nanostructurization. After 5-h oxidation, a rather uniform array of nanoneedles oriented normally to the sample surface appears having the density of the order of $10^{10}-10^{11} \mathrm{~cm}^{-2}$, the height of certain nanoneedles being close to $20 \mathrm{~nm}$. A change of the oxidation time results in transformation of surface topology. Optimization of technological conditions for oxidation can be used for nanodimensional ordering the oxide surface.

\section{References}

1. S.M. Sze, Physics of Semiconductor Devices. John Wiley \& Sons, New York, 2-nd ed., 1981.

2. A.-L. Barabasi, Self-assembled island formation in heteroepitaxial growth // Appl. Phys. Lett. 70(19), p. 2565-2567 (1997).

3. Z.D. Kovalyuk, V.M. Katerynchuk, A.I. Savchuk, and O.S. Lytvyn, Topology of GaSe oxidized crystals // Superlattices and Microstructures, 44(45), p. 416-419 (2008).

4. T. Ikari, S. Shigetomi, K. Nashimoto, Crystal structure and Raman spectra of InSe // Phys. status solidi (b), 111(2), p. 477-481 (1982).. 